\title{
Efecto de las condiciones de corte de un láser de Nd:YAG sobre la estructura y microcomposición de la superficie de aceros inoxidables
}

\author{
A. Ramírez ${ }^{(*)}$, J. Pascual ${ }^{(*)}$, J. Laserna ${ }^{(* *)}$, M.V. Moya ${ }^{(*)}$, J. Zapatero ${ }^{(*)}$ y T. Fernández ${ }^{(*)}$
}

Resumen Se presenta un estudio de la influencia de los parámetros de procesado láser (distribución temporal, distribución espacial y velocidad) en la calidad de acabado y microestructura de aceros inoxidables austeníticos. Se ha utilizado un láser Nd:YAG con nitrógeno como gas de protección, analizándose los resultados mediante SEM, microsonda electrónica y análisis metalográfico. Para una distribución espacial fija (10\%), y una frecuencia de $500 \mathrm{~Hz}$, el aumento de la distribución temporal produce mayor potencia, lo que permite trabajar a velocidades más altas. La calidad del acabado aumenta al hacerlo la velocidad de corte. El corte de acero 304 con láser a velocidades superiores a $0,0033 \mathrm{~m} / \mathrm{s}$ y potencias inferiores a $1.000 \mathrm{~W}$ usando nitrógeno a 18 bar no produce modificaciones apreciables en la estructura.

Palabras clave: Láser. Aceros. Corte. Procesado. Metalografía.

\section{Effect of the cutting conditions with a Nd:YAG laser on stainless steels surface structure and microcomposition}

\begin{abstract}
A study is presented of the effect of laser parameters (time distribution, spatial distribution and speed) on both the finishing quality of austenitic stainless steel and its microstructure. An Nd-YAG laser with nitrogen as protection gas has been used. The samples were studied by SEM, metallography and microprobe analysis. At $500 \mathrm{~Hz}$ and $10 \%$ of the spatial distribution, any increase of the time distribution produces higher power; this effect allows working at higher speed. Quality increases with the cutting speed. The laser cutting of 304 stainless steel using speeds higher than 200 $\mathrm{mm} / \mathrm{min}$ and power lower than $1,000 \mathrm{~W}$ and a nitrogen flow of 18 bar of pressure do not produce significative changes in the structure.
\end{abstract}

Keywords: Laser. Steel. Cutting. Processed. Metallography.

\section{INTRODUCCIÓN}

La gran profusión que han alcanzado los sistemas de procesado de materiales mediante láser, es consecuencia de su capacidad para proporcionar un flujo elevado de energía además de la facilidad de ser dirigidos, lo que nos permite acoplarlo a sistemas de fabricación asistidos por ordenador.

(*) Dpto de Ingeniería Civil, de Materiales y Fabricación. E.T.S.I.I. Univ. de Málaga. Plaza del Ejido s/n. 29013Málaga (España).

(**) Dpto. de Química Analítica. Fac. de Ciencias. Campus de Teatinos. Univ. de Málaga (España).
La aplicación industrial del láser presenta una serie de ventajas sobre los sistemas convencionales, entre las que podemos destacar:

- Posibilidad de concentrar la energía en una zona muy pequeña sin afectar al resto del material.

- Ausencia de vibraciones al interaccionar el haz con la materia con lo que no es necesario el anclaje del material que se está procesando.

- Fácil manipulación de la radiación.

- Posibilidad de acople a un proceso automático.

El corte con láser es un proceso de absorción de radiación electromagnética en el ámbito del IR, 
produciéndose el calentamiento del material receptor hasta la vaporización a lo largo de la zona de corte (1). Además del generador y del sistema óptico, se usa un gas a presión, concéntrico con el haz, para purgar el material fundido y vaporizado. Este incide a presión sobre la superficie de corte desplazando el material fundido y vaporizado y protegiendo así al sistema de la acción del material a alta temperatura y de la posible reflexión del haz en el plasma formado por la acción del haz sobre el material. Este gas puede ser inerte o reactivo. El gas inerte se usa para proteger la zona de corte de la oxidación o cuando hay peligro de explosión, en el caso de materiales inflamables. Los gases reactivos producen reacciones exotérmicas, con lo que permiten aumentar de forma notable la velocidad de proceso, aunque se produce la oxidación del material (2).

Como parámetros a tener en cuenta en el procesado láser, se pueden citar: potencia suministrada por el láser, distribución espacial y temporal de energía, distancia focal, longitud de onda, polarización, divergencia y sección transversal del haz. Las características y comportamiento del material son igualmente determinantes, así habrá que considerar: absorción del haz, conductividad térmica, densidad, calor específico, temperaturas de cambio de fase y calor latente asociado a cada cambio de fase $\mathrm{y}$, por último, las dimensiones del material (3). Aunque tampoco son descartables otros factores de la zona de actuación, como son área de fusión, porosidad y defectos de superficie (4).

Actualmente, el láser más utilizado en la industria es el de $\mathrm{CO}_{2}$, aunque los láseres de $\mathrm{Nd}$ (Nd:YAG y vidrio de $\mathrm{Nd}$ ) van implantándose progresivamente, ya que son aplicables a la mayoría de los materiales en los que se trabaja con $\mathrm{CO}_{2}$, aunque la menor potencia disponible rebaje la velocidad de corte y el espesor máximo del material.

La luz generada por un YAG tiene una longitud de onda de $1,06 \mathrm{~mm}$, mientras que la del $\mathrm{CO}_{2}$ es 10 veces mayor. El emitir a una frecuencia superior tiene una serie de ventajas como son:

- Los vidrios suelen ser transparentes a esta longitud de onda, lo que permite focalizar el haz y proteger el sistema con este material, permitiendo guiar el haz a través de fibra óptica.

- El diámetro del punto focal es proporcional a la longitud de onda, siendo el del Nd:YAG de 10,6 $\mathrm{mm}$, aproximadamente $1 / 10$ del de $\mathrm{CO}_{2}$.

- La absorción de los metales a la radiación infrarroja aumenta al disminuir $\lambda$ por lo que, para materiales altamente refractarios, el Nd:YAG da mejores resultados.

Pese a las ventajas derivadas de trabajar a mayor frecuencia, el Nd:YAG todavía tiene una implantación reducida, debido a que la potencia disponible es mucho menor que en el $\mathrm{CO}_{2}$ (quedando por debajo de los $2.000 \mathrm{~W}$ ). Consecuentemente, la velocidad de corte es menor y, por último, no es aplicable al corte de polímeros transparentes.

La posibilidad de guiar el haz por fibra óptica incrementa notablemente su aplicación al procesado de piezas complicadas y facilita la automatización de procesos. No obstante, el recorrido del haz por la fibra óptica produce pérdidas de intensidad con la consiguiente disminución de potencia (3).

En el corte de metales y aleaciones, el gas utilizado está en función de los requerimientos que se tengan acerca del material y pieza a fabricar. Así, cuando lo que interesa es cortar grandes espesores o alcanzar velocidades altas, se utiliza oxígeno, que suministra calor adicional (hasta un $60 \%$ ) como consecuencia de la reacción con los metales, mientras que para obtener acabados de alta calidad se utiliza un gas inerte de alto poder refrigerante (generalmente nitrógeno) (5).

La velocidad de corte determina el espesor máximo de trabajo y el acabado. Los parámetros que controlan el gas de protección (caudal y presión) intervienen de forma directa en el acabado. Sin embargo, estas variables también rigen los procesos de evaporación-arrastre y condensación de los metales que forman el acero inoxidable, por lo que su incidencia en el proceso de corte es múltiple (6).

\section{MÉTODOS Y MATERIALES}

\subsection{Métodos}

Se ha utilizado una fuente láser de Nd:YAG (ROFIN-SINAR), con una potencia media de 1.200 W y una banda de frecuencias de trabajo de 1 a 500 $\mathrm{Hz}$, con duraciones de pulso programables entre 0,3 y $20 \mathrm{~ms}$, perfilable en 20 sectores. La energía máxima de pulso es de $120 \mathrm{~J}$, lo que permite gran capacidad de penetración en el material. El equipo se integra en un sistema MC7 de 5 ejes CNC SINUMERIK.

La frecuencia de trabajo ha sido de $500 \mathrm{~Hz}$, manteniendo una energía que en ningún caso ha superado $1 \mathrm{~J}$. Para conseguir fijar estos parámetros, se ha trabajado con un único sector con una distribución espacial de energía del $10 \%$. Se ha variado la distribución temporal de energía de 0,4 a $0,9 \mathrm{~ms}$, obteniendo potencias medias entre 200 y $800 \mathrm{~W}$. Esta variación de la potencia ocasiona una variación en la velocidad de corte, que aumenta a medida que aumenta la potencia (Tabla I).

Como gas de aporte se ha utilizado nitrógeno a una presión de 18 bares, debido a que el uso de un gas inerte a alta presión mejora considerablemente el acabado del borde de corte (3). Se ha descartado el uso de oxígeno, aunque permite 
TABLA I.- Relación potencia-distribución temporalvelocidad de corte en un láser $\mathrm{Nd}$ :Yag.

TABLE I.- Relationship power-time distributioncutting speed in the Nd-YAG laser.

\begin{tabular}{|c|c|c|}
\hline $\begin{array}{c}\text { Distribución } \\
\text { temporal } \\
\text { de energía }(\mathrm{ms})\end{array}$ & $\begin{array}{c}\text { Potencia } \\
\text { media } \\
\text { (W) }\end{array}$ & $\begin{array}{c}\text { Velocidad } \\
\mathrm{m} / \mathrm{s}\end{array}$ \\
\hline 0,4 & 200 & 0,0033 \\
0,5 & 370 & 0,0050 \\
0,6 & 480 & 0,0150 \\
0,7 & 570 & 0,0200 \\
0,8 & 670 & 0,0250 \\
0,9 & 740 & 0,0266 \\
\hline
\end{tabular}

trabajar a velocidades superiores, por elevación local de la temperatura por reacciones de oxidación (4), ya que su uso conduce a un acabado que precisa un mecanizado posterior.

El análisis micromorfológico de las muestras se ha realizado con un microscopio electrónico Jeol 6400 utilizando tensiones de aceleración de 10 y 20 $\mathrm{kV} \mathrm{y}$, analizando las imágenes de electrones secundarios y retrodispersados obtenidas. Este microscopio está equipado con microsonda electrónica de dispersión de energías con detector de ligeros.

Para optimizar la velocidad de corte, se ha tenido en cuenta el acabado final del borde de la muestra atendiendo a alguna de las siguientes características:

- Anchura de corte de algunas centenas de mm.

- Relieve menor de $1,5^{\circ}$.

- Estado de oxidación de las caras.

- Ausencia de rebabas y adherencia.

- Presencia de una zona afectada térmicamente.

- Homogeneidad del corte en cualquiera de las direcciones.

- Idéntico estado en cualquiera de las caras.

- Ausencia de quemaduras.

La preparación para el estudio metalográfico se ha realizado con pulido y posterior ataque electrolítico usando una disolución de $\mathrm{HNO}_{3}$.

\subsection{Material}

El material utilizado para este estudio ha sido un acero ANSI 304, también conocido como acero austenítico $18-8$, con un espesor de $10^{-3} \mathrm{~m}$ y acabado mate.

\section{RESULTADOS EXPERIMENTALES Y DISCUSIÓN}

En estudio metalográfico del acero (Fig. 1) se observa la estructura austenítica típica con falta de

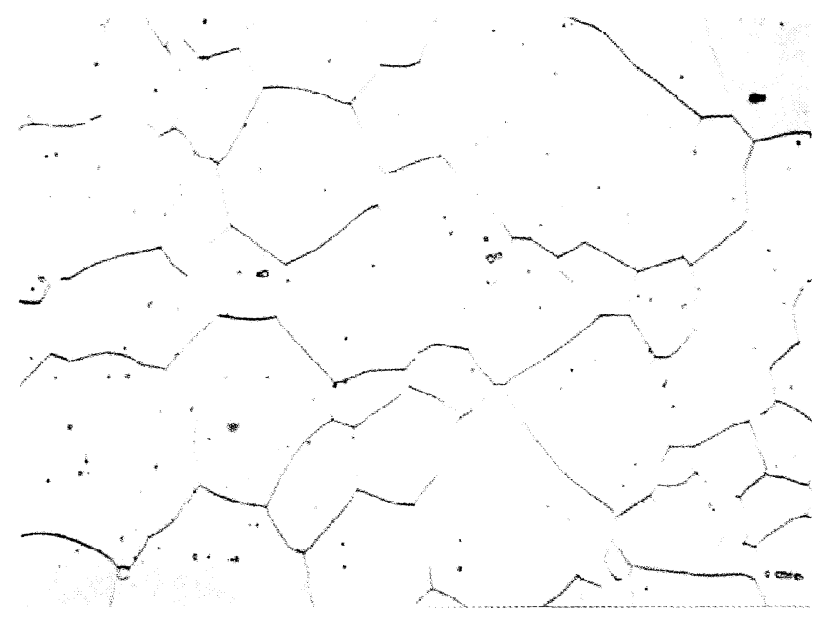

FIG. 1.- Preparación metalográfica de acero ANSI 304.

FIG. 1.- Metallographic sample of stainless steel ANSI 304. X1000

homogeneidad en el tamaño de grano. Este hecho no permite una cuantificación de las variaciones observadas. No obstante, puede apreciarse una disminución del tamaño de grano en las zonas próximas al corte (Fig. 2).

Cuando la velocidad de corte se mantiene en valores bajos, $<0,0166 \mathrm{~m} / \mathrm{s}$, y la potencia entre 200 y $500 \mathrm{~W}$, se produce la inclusión de nódulos esféricos en la zona externa del corte y en su entorno (Fig. 3), cuya presencia se atribuye a la solificación de metales evaporados y de sus óxidos que quedan ocluidos formando compuestos metal-óxido, como se aprecia en el espectro de la figura 4. A estas velocidades, se produce una banda de espesor variable entre 1 y $3 \cdot 10^{-5} \mathrm{~m}$, en la que los granos son menores $\left(<5 \cdot 10^{-6} \mathrm{~m}\right)$ que en la zona contigua al cordón como muestra la figura 5 , que presenta la

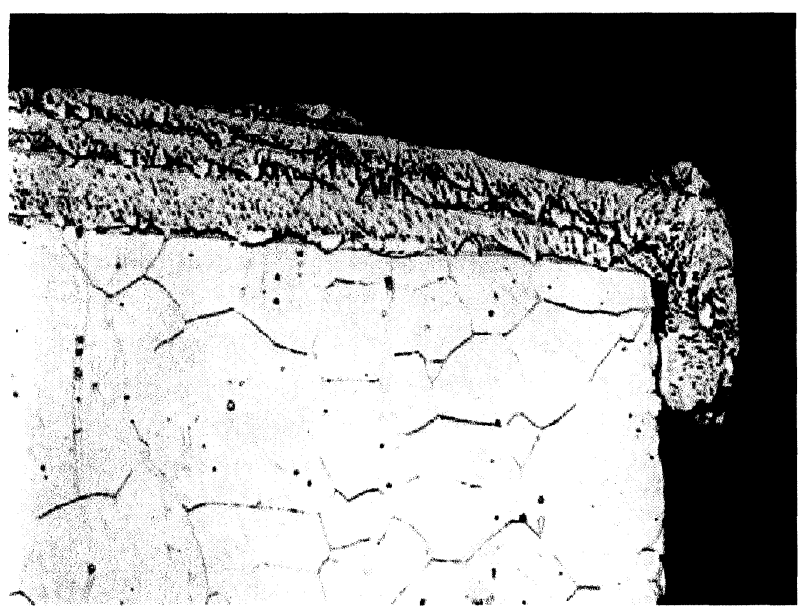

FIG. 2.- Imagen metalográfica del acero ANSI 304 en las proximidades del corte láser.

FIG. 2.- Metallographic image of stainless steel ANSI 304 near to laser cutting. X1000 


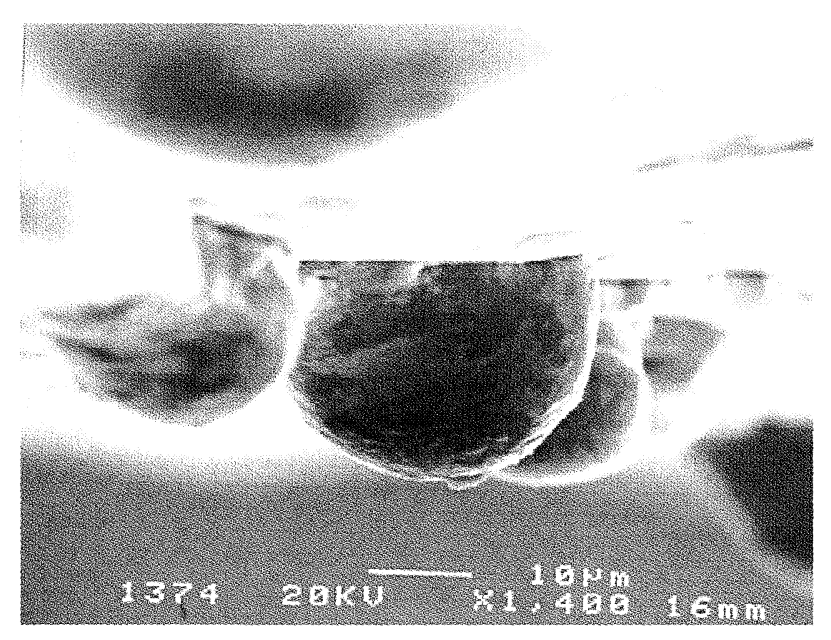

Fig. 3.- Micrografía de las inclusiones de nódulos esféricos en el fundido de corte.

FIG. 3.- Micrography of sphericals niddles inclusions on melting by cutting.

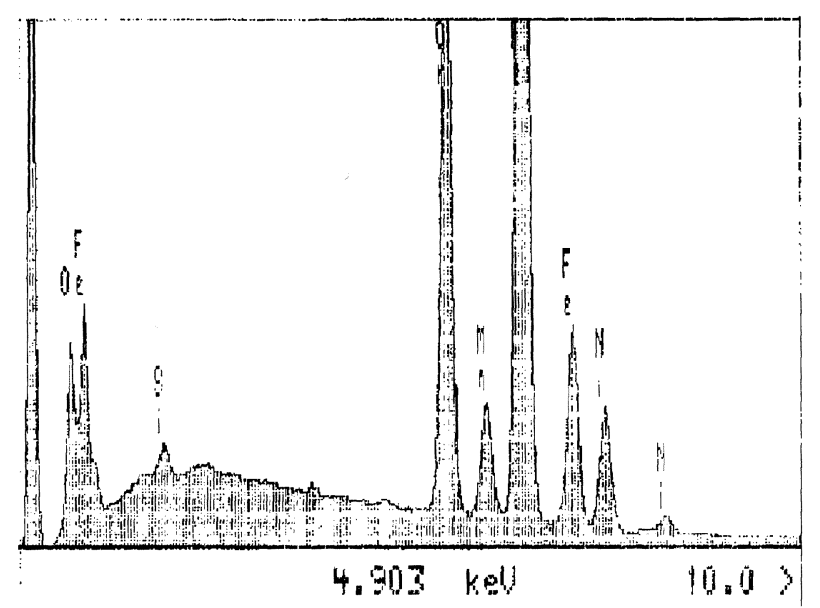

FIG. 4.- Espectro de emisión de rayos $\mathrm{X}$ de los nódulos esféricos.

FIG. 4.-X-ray spectrum emision of spherical niddles.

imagen de electrones retrodispersados. En la cara posterior, no se observa esta banda, por lo que su presencia puede atribuirse a sobrecalientamiento local. Por otro lado, en la cara posterior, la discontinuidad del haz provoca gradientes de temperatura en el fundido, variando su viscosidad y provocando cordones laterales discontinuos de espesores menores de $10^{-5} \mathrm{~m}$ y acumulaciones en las crestas (Fig. 6). La figura 7 corresponde al espectro de emisión de rayos X de estos fundidos; en ellos, se aprecia el descenso del contenido de hierro en favor del manganeso, cromo y níquel, siendo destacable la presencia local de silicio. La discontinuidad del fundido se manifiesta en las zonas en las que es inexistente (Fig. 8). Su falta parece deberse a arrastre mecánico, consecuencia de su mala adherencia

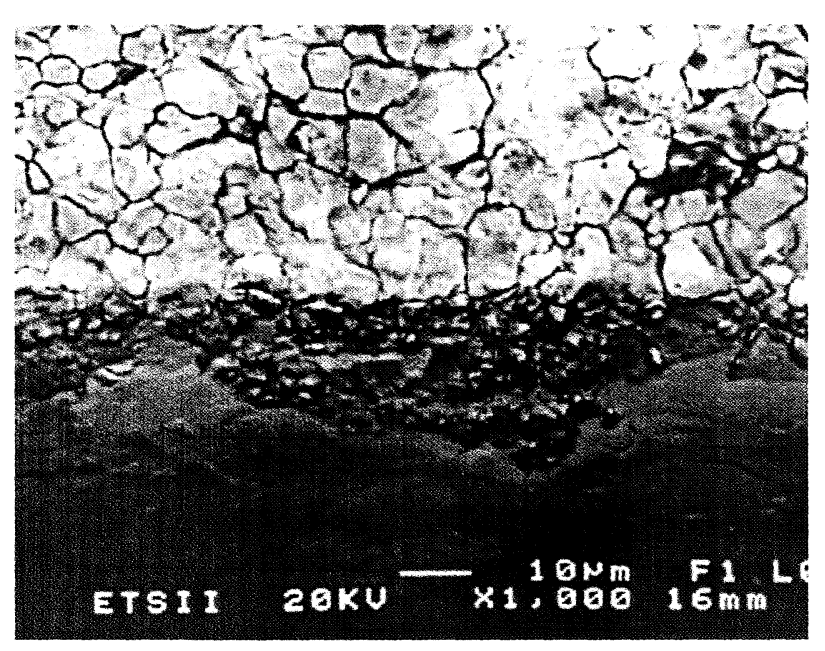

FIG. 5.- Banda contigua al corte afectada térmicamente de menor tamaño de grano que el acero sin tratar.

FIG. 5.- Affected thermically zone near to the cut that it show less grain dimension than the stainless steel not heated one.

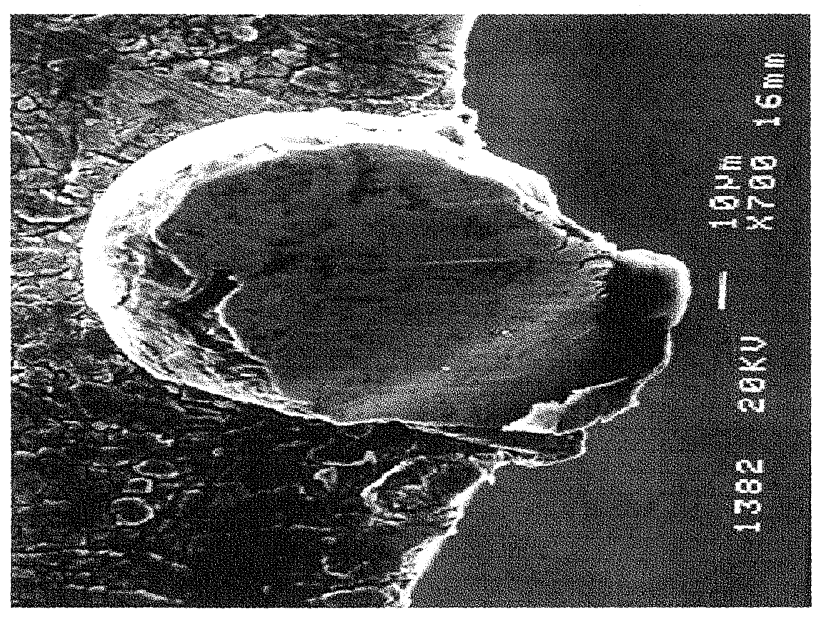

Fig. 6.- Cara de salida del haz. Acumulación de fundido en las crestas.

FIG. 6.- Outside face of the laser beam. It show melt accumulation in the edge.

al acero base. Las imágenes de rayos X (Fig. 9) informan sobre el acero base que ha quedado después de desprenderse el fundido; así, este queda totalmente desprovisto de oxígeno en superficie, por lo que parece que el fundido en contacto con el acero base ha disuelto el óxido superficial del acero base incorporándolo a un compuesto metal-óxido que se sitúa en la interfase. La formación local de $\mathrm{SiO}_{2}$ debe facilitar el desprendimiento del fundido. A velocidades superiores, no se observa esta banda, dado que la temperatura alcanzada es menor.

Las figuras 10 y 11 presentan las secciones transversales de la zona de entrada y salida del haz, 


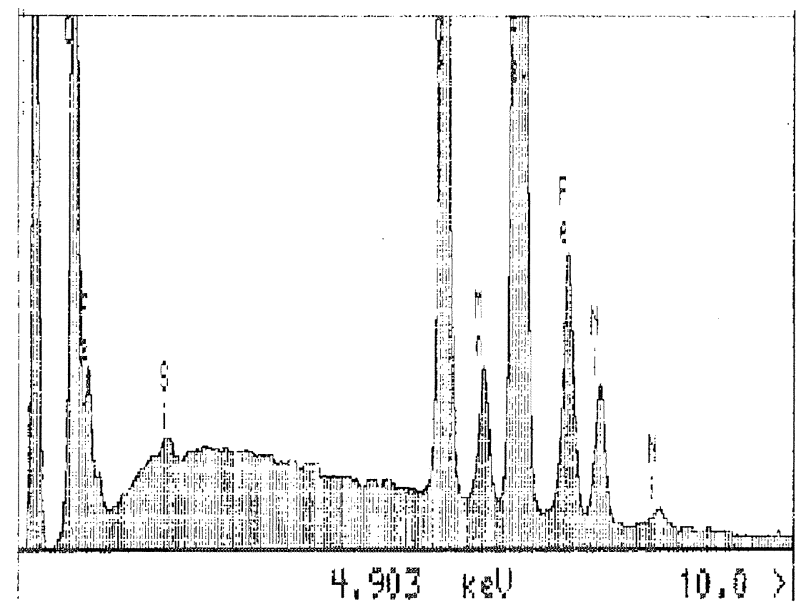

Fig. 7.- Espectro de emisión de rayos $\mathrm{X}$ de una parte del fundido.

FIG. 7.-X-ray spectrum emision of a piece of melt.

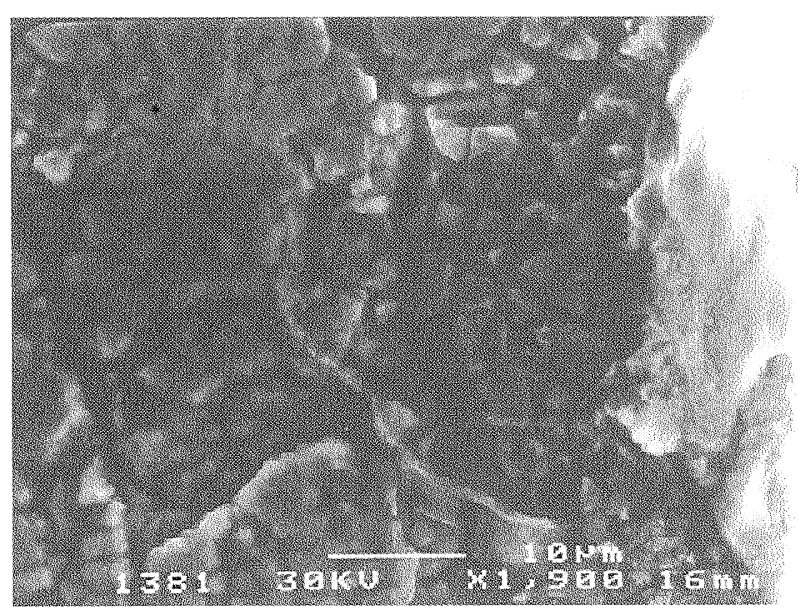

FIG. 8.- Imagen SEM de una zona en la que se ha arrancado el fundido.

FIG. 8.- SEM image of a zone which the melt has been removed.

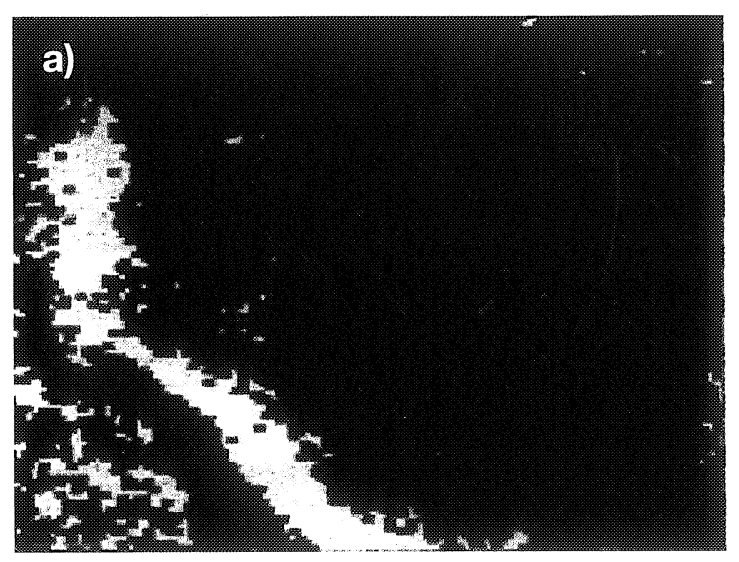

FIG. 9.- Imágenes de rayos X: Zona de la foto anterior: a) $\mathrm{O}_{2}$; b) Si; c) $\mathrm{Cr}$; d) Fe; e) $\mathrm{Ni}$

FIG. 9.-X-ray images: The last photo zone: a) $\mathrm{O}_{2}$; b) $\mathrm{Si}$; c) $\mathrm{Cr}$; d) $\mathrm{Fe}$; e) $\mathrm{Ni}$

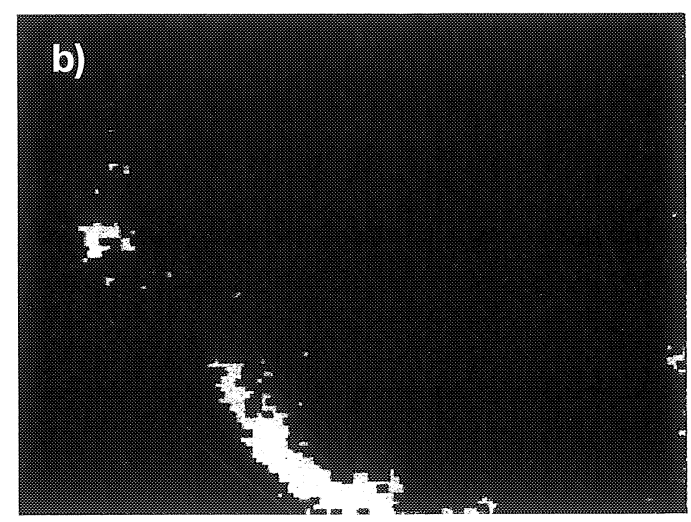

c)
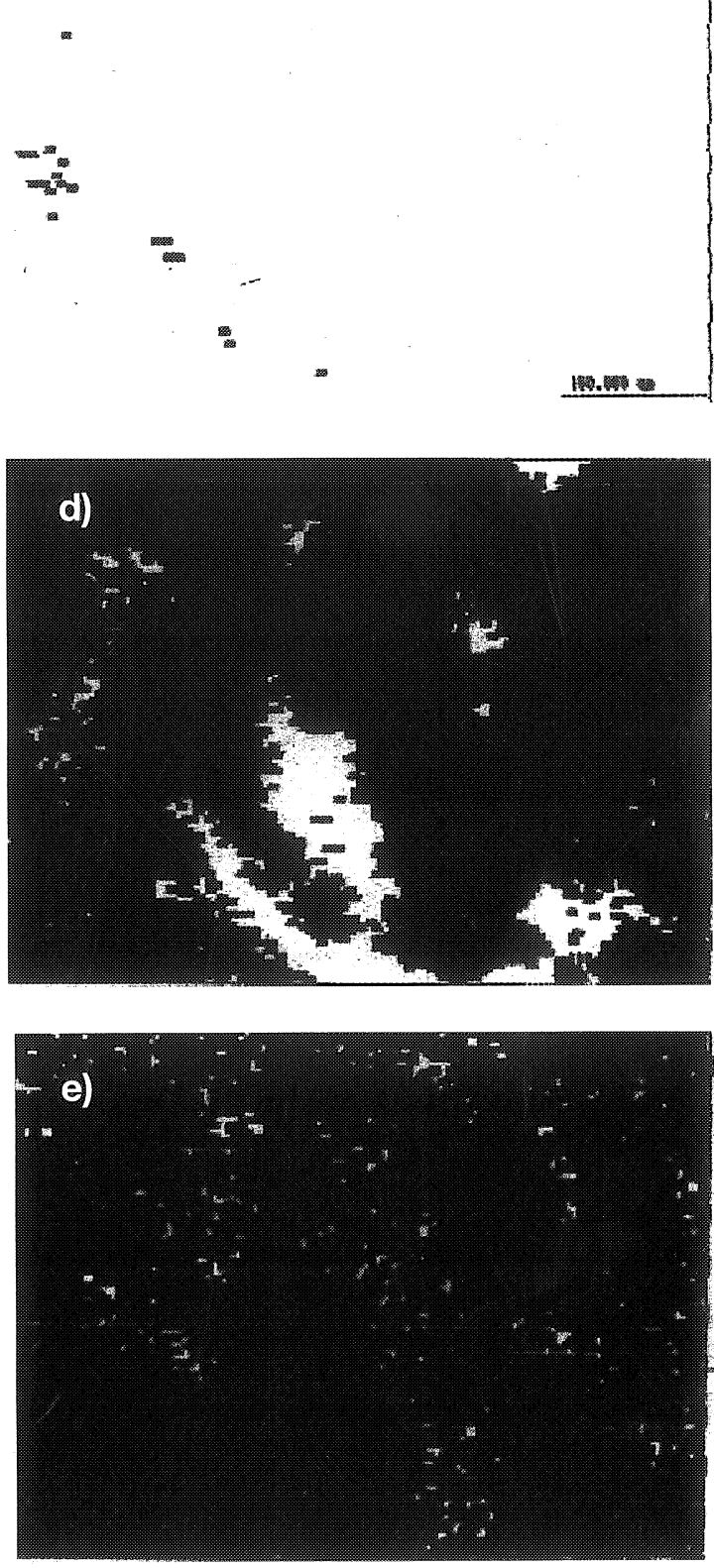

en cortes con velocidad de corte inferior a 0,0166 $\mathrm{m} / \mathrm{s}$. Puede observarse el aumento de espesor del cordón de fundido al descender. Este puede 


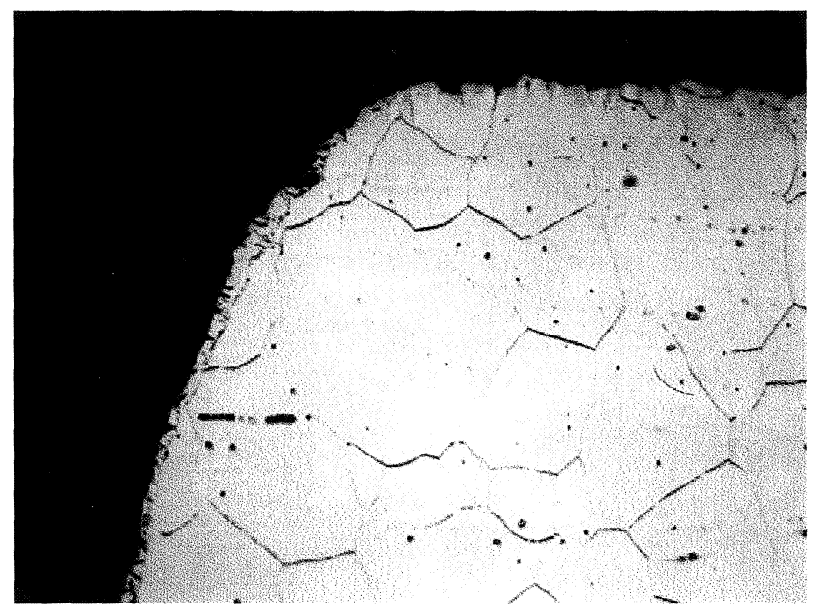

Fig. 10.- Sección transversal de la zona de entrada del haz a velocidad de corte inferior a $0,0166 \mathrm{~m} / \mathrm{s}$

FIG. 10. - Transversal section of the beam in zone at cutting speed lower to $0,0166 \mathrm{~m} / \mathrm{s}$

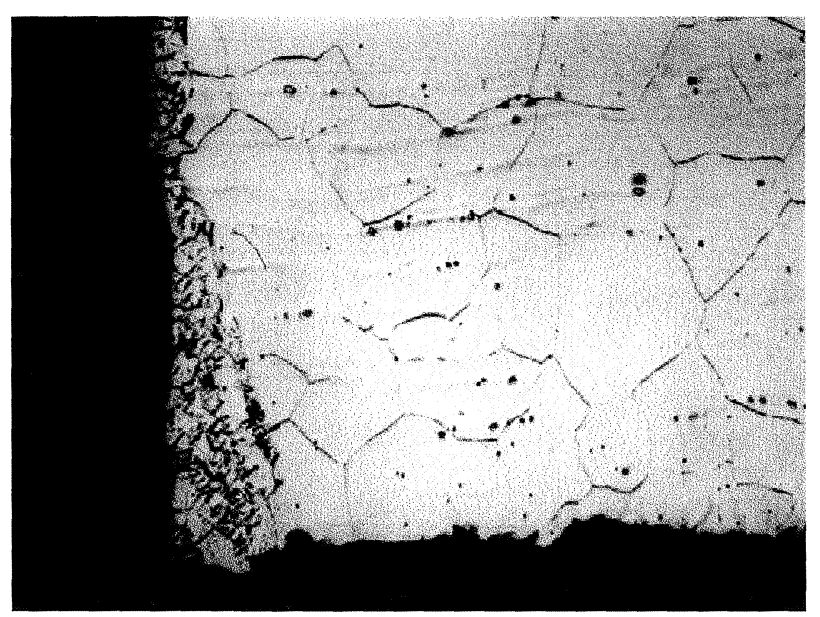

FIG. 11.- Sección transversal de la zona de salida del haz a velocidad de corte inferior a $0,0166 \mathrm{~m} / \mathrm{s}$

FIG. 11.- Transversal section of the beam out zone at cutting speed lower to $0,0166 \mathrm{~m} / \mathrm{s}$

atribuirse al aumento de viscosidad que experimenta el fundido a causa del enfriamiento. En contraposición, al aumentar la velocidad de corte, los espesores de fundido son menores y más homogéneos, como consecuencia de mantenerse a temperaturas superiores (Figs. 12 y 13).

Así mismo, a velocidades súperiores a 0,0166 $\mathrm{m} / \mathrm{s}$, se producen salpicaduras, en la cara posterior, en forma de pequeñas esferas de óxidos (Fig.14) que llegan hasta las $2 \cdot 10^{-4} \mathrm{~m}$ del borde de corte. Estas salpicaduras se producen por un efecto de atomización del fundido por el nitrógeno, produciéndose su oxidación por contacto con el oxígeno del aire presente en la zona por depresión local.

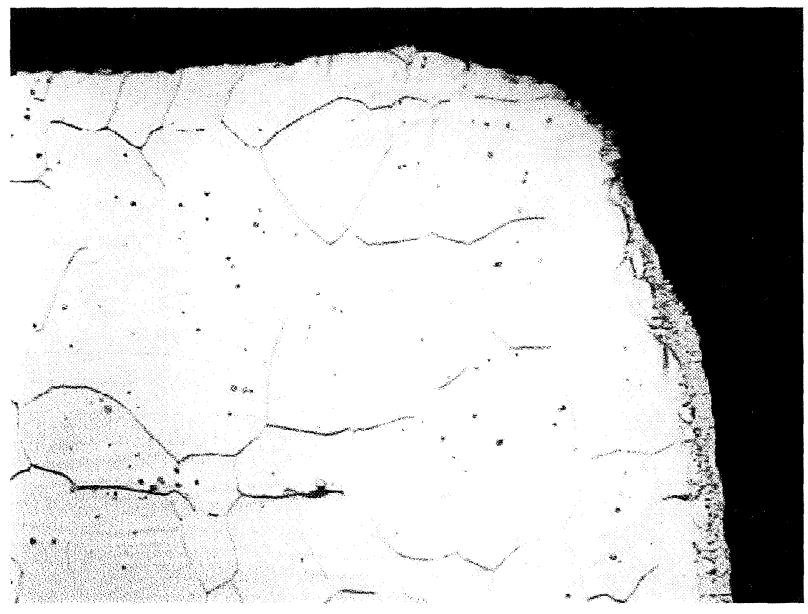

FIG. 12.- Sección transversal de la zona de entrada del haz a velocidad de corte superior a $0,0166 \mathrm{~m} / \mathrm{s}$

FIG. 12.- Transversal section of the beam in zone at cutting speed up to $0,0166 \mathrm{~m} / \mathrm{s}$

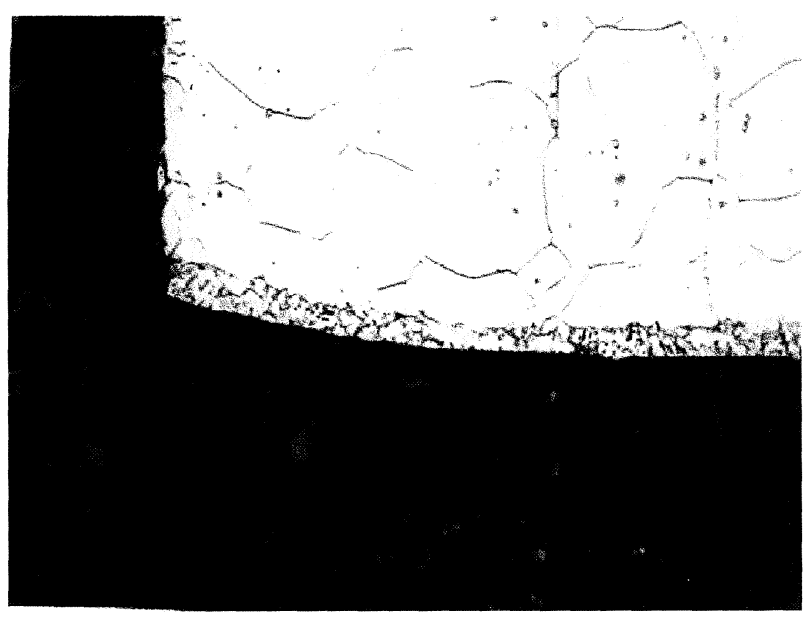

FIG. 13.- Sección transversal de la zona de salida del haz a velocidad de corte superior a $0,0166 \mathrm{~m} / \mathrm{s}$

FIG. 13.- Transversal section of the beam out zone at cutting speed up to $0,0166 \mathrm{~m} / \mathrm{s}$

\section{CONCLUSIONES}

$\mathrm{Al}$ aumentar la distribución temporal de energía, para distribuciones espaciales del orden del $10 \%$, permanece constante la frecuencia $(500 \mathrm{~Hz})$ produciéndose una elevación de la potencia disponible, lo que permite la utilización de mayores velocidades de corte.

A frecuencias de $500 \mathrm{~Hz}$. y potencias del orden de $800 \mathrm{~W}$, un aumento de la velocidad hasta los $0,0266 \mathrm{~m} / \mathrm{s}$ produce mejor calidad en el acabado del corte utilizando nitrógeno como gas de protección.

El corte de acero ANSI 304 con láser Nd-YAG, en las condiciones descritas, no produce cambios estructurales significativos que puedan modificar el comportamiento del material, incluso en las proximidades del corte. 


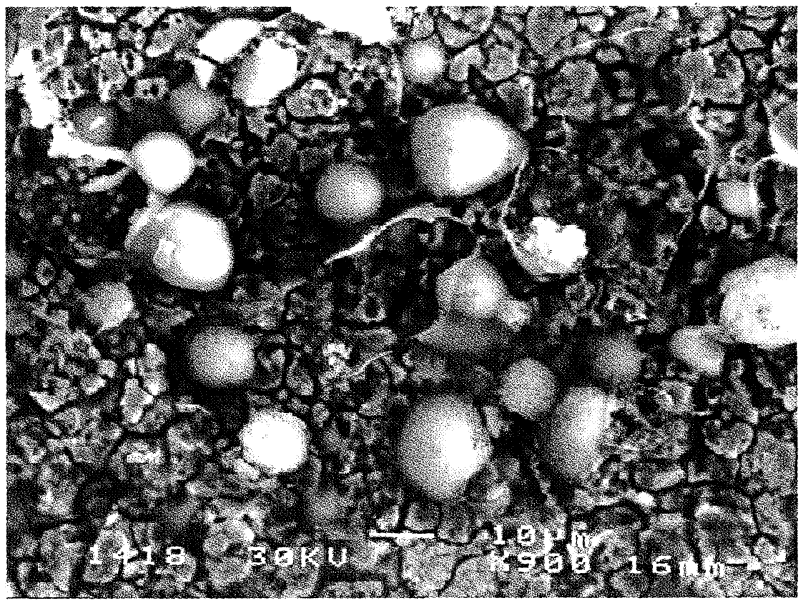

FIG. 14.- Imagen SEM de salpicaduras de óxidos, cara posterior a velocidades superiores a $0,0166 \mathrm{~m} / \mathrm{s}$.

FIG. 14. - SEM images of oxide splash, at the backside at cutting speed up to $0,0166 \mathrm{~m} / \mathrm{s}$

\section{REFERENCIAS}

(1) OHSE, W. The Industrial Laser Annual Handbook. Belforte. D, Levitt. M. Penn Well Books. Tulsa, (Oklahoma, EE.UU.). 1990: 30-48

(2) Ion, J.C. The Industrial Laser Handbook. Belforte. D., Levitt. M., Springer-Verlag. 1993: 39-45.

(3) Ivarson. A, Powell. J. et al. Proc. ICAlEO`91 Laser Institute of America. San José (Cal, EE.UU.) 1991: 211 219.

(4) Chen. S.L. y Steen W.M. Proc. ICALEO’91. Laser Institute of America. San José (Cal, EE.UU.) 1991: 221230.

(5) Liu, JT., Weckman. D.C. y KerR H.W. Metall Trans. B. Process Metallurgy. 24, 1993: 1065-1076.

(6) LiPPOLD. J.C. Weld. J. 73, 1994: S129-S139. 